\title{
Long-term Survival Advantage for Recurrent Ovarian Cancer Patients Receiving Pegylated Liposomal Doxorubicin
}

\author{
a report by \\ Dr Alan N Gordon
}

Clinical Professor of Obstetrical Gynecology, University of Arizona School of Medicine

DOI: $10.17925 / O H R .2005 .00 .00 .83$

Ovarian cancer remains the leading cause of death from gynaecologic malignancies. Although endometrial cancer occurs more frequently, the vast majority of patients who develop this disease present with post-menopausal or other irregular bleeding problems and are usually diagnosed with early-stage disease and experience long survival. In contrast, the presenting signs and symptoms of ovarian cancer are non-specific and almost $75 \%$ of patients already have extensive abdominal disease at the time of diagnosis (stage IIIC and IV). The failure to be able to detect ovarian cancer at earlier stages has led to it being labelled 'the silent killer'.

The use of extensive debulking procedures, which may include resections of the gastrointestinal (GI) or urinary tracts, can decrease the tumour burden for cytotoxic chemotherapy and help to prolong survival. The addition of taxanes to platinum-based therapy have led to significant improvement in both progression-free and overall survival in patients with either optimally reduced or suboptimally reduced advanced-stage disease.

Currently, ovarian cancer patients are living longer and as the disease tends to remain within the peritoneal cavity without affecting vital organs, patients may maintain a good performance status for a long time. Unfortunately, disease usually relapses. Relapse may be heralded by elevation of CA-125 and eventually symptoms and evidence of disease will appear, leading to treatment of recurrent disease. With patients living longer and receiving therapy for longer periods of time, there is a need for agents that are well tolerated, lacking of cumulative toxicity and able to maintain quality of life rather than worsen it due to unintended side effects.

Treatment at time of relapse has been guided by the interval from the patients' prior platinum exposure. Those patients who had demonstrated progression of disease while on platinum-based therapy, or relapsed within six months from their last platinum therapy, were thought to be platinum-resistant and should be treated with other non-cross-resistant agents. Patients who had initially responded and demonstrated clinical progression more than six months after their last platinum therapy were thought to have potentially platinum-sensitive disease and would be re-treated with platinum agents.

The probability of responding to re-treatment increases with time from the last platinum exposure. After a platinum-free interval of two years, response rates may approach $60 \%$, which is similar to initial response rates in untreated disease. Although response rates at earlier intervals were lower, prior to the late 1990s there were no agents that were thought to have significant activity in relapsed ovarian cancer and platinum-based therapy was the standard.

Paclitaxel did exhibit activity in recurrent ovarian cancer but, based on several studies, quickly moved into first-line therapy as part of a taxane and platinum-based combination. Then, several agents in phase II trials showed promising activity in relapsed ovarian cancer. A phase III trial, comparing topotecan with paclitaxel, showed statistically equivalent response rates and survival data in paclitaxel naïve patients. Based largely on this data, topotecan was approved for use in relapsed ovarian cancer by the US Food and Drug Administration (FDA). Phase II trials with pegylated liposomal doxorubicin (PLD) that had been underway had shown activity for PLD in platinum refractory ovarian cancer. It was then decided to proceed with a phase III trial comparing PLD with topotecan.

The results of this phase III trial were initially reported in 2001. There were 474 patients enrolled from 104 sites from around the world. Patients were stratified upon enrollment based on platinum sensitivity or resistance and on the presence or absence of bulky disease. There was no difference in response rates in the overall or any subgroup analysis. In the survival data that were available, there was no difference in progression-free or overall survival for the entire group. However, there was a significant improvement in progression-free survival and an even greater 
Figure I: PLD Relative to ICON 4

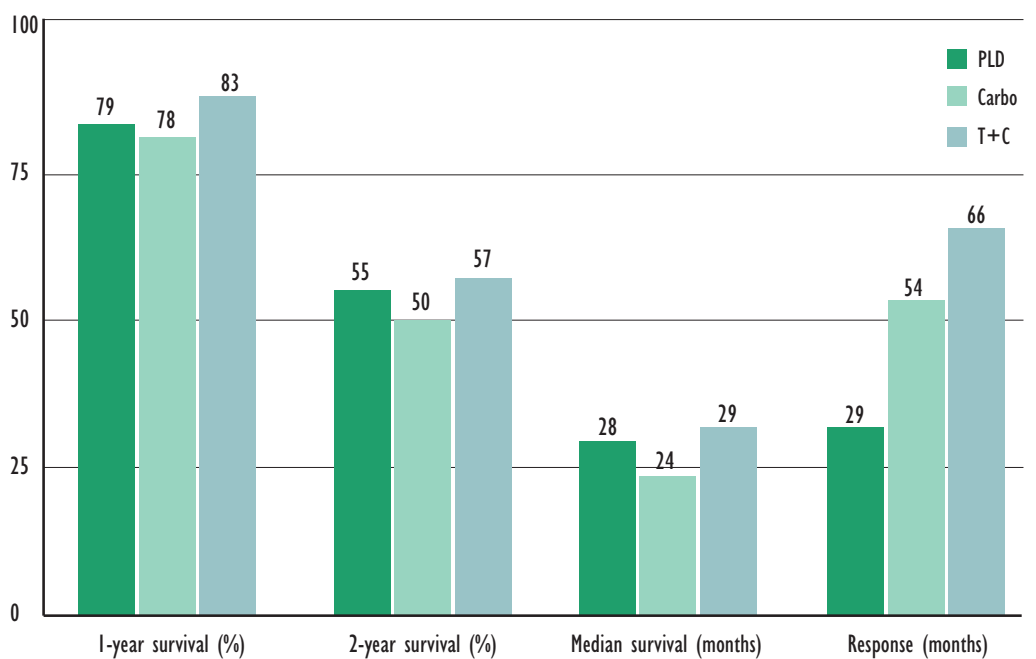

Table I: Patient Demographics

\begin{tabular}{llll} 
& ICON 4 & 30-49 & AGO \\
\hline$>12$ & $75 \%$ & $44 \%$ & $60 \%$ \\
months & & & \\
\hline $\begin{array}{l}\text { No Prior } \\
\text { Taxane }\end{array}$ & $58 \%$ & $26 \%$ & $32 \%$ \\
\hline
\end{tabular}

improvement in overall survival for the platinumsensitive patients initially treated with PLD. There was a large number of censored events as many of the patients had not progressed or died at the time of the report. After more than $90 \%$ of the patients had recurred or died, a planned updated analysis was performed to re-evaluate the survival data. in death for initial treatment with PLD that was significant at $\mathrm{p}=0.017$.

What does this mean for patients with platinum refractory disease? Currently, there are three randomised phase III trials of single agents in platinum-resistant disease. All agents have shown similar activity whether measured by response or survival data. No single agent has been shown to be superior to another; therefore, in patients with platinum-refractory disease, the choice of agent should be based on toxicity and quality of life criteria. The lack of alopecia, minimal nausea/ vomiting, minimal myelosuppression and ease of administration, with a lack of cumulative toxicity, would favour PLD as an initial choice for many patients with platinum-refractory disease. Some authors have shown activity for the combination of gemcitabene and cisplatinum in patients with resistant disease; however, there is significant toxicity with this regimen and a randomised comparison has not been performed.

In patients with platinum-sensitive disease, several phase III trials have compared non-platinum agents; however, there are no available data comparing platinum with a non-platinum agent in platinum-sensitive disease. Response rates for all agents in all the randomised trials are about 30\%, which is similar to the response rates reported for platinum re-treatment in the 12 - to 24-month platinum-free interval. In view of the prolonged progression-free and overall survival seen with PLD relative to topotecan, response rates may not

\section{With long-term follow-up, overall survival for all patients now showed a statistically significant improvement for initial therapy with PLD.}

With long-term follow-up, overall survival for all patients now showed a statistically significant improvement for initial therapy with PLD. Although the median survivals were similar at 63 weeks for PLD versus 60 weeks for topotecan, there was an $18 \%$ risk reduction in death for all patients that was significant, with $p=0.032$. There were still no significant differences in the patients with platinum-resistant disease. However, in the platinum-sensitive group, patients initially treated with PLD did significantly better. The median survival was 27 months for initial treatment with PLD versus 17.5 months for initial treatment with topotecan. Overall, there was a 30\% risk reduction be the best measure of activity in this group of patients. It is still not possible to compare a nonplatinum agent with a platinum analogue; however, based on the improved progression-free and overall survival with PLD, this should be the first choice of the non-platinum agents.

Recently, two randomised trials have compared single-agent carboplatin with carboplatin combination in platinum-sensitive patients. ICON 4 used paclitaxel as the second agent and demonstrated significantly improved response rates, progression-free survival and overall survival in the patients receiving the combination. The AGO- 


\title{
Confirmed: CAELYX ${ }^{\mathrm{Tm}}$ significantly prolongs survival compared with topotecan'
}

\section{LONG - TERM ANALYSIS REINFORCES \\ CAELYX ${ }^{m}$ SUPERIORITY',}

- $\quad \mathbf{8} \%$ risk reduction in death for all patients with

CAELYX" vs topotecan: $P=0.032(N=474)$

\section{"...the only non-platinum agent to demonstrate a progression-free and overall survival advantage in platinum-sensitive disease...'}

\author{
UPDATED NICE* GUIDELINES RECOMMEND \\ EARLIER USE OF CAELYX ${ }^{m}$ \\ - The only single agent option for the newly recognized category \\ of partial platinum sensitivity ${ }^{3}$ \\ - Tumor recurrence at 6 to 12 months following initial \\ platinum-based treatment
}

- Also recommended for platinum-resistant or platinum-refractory advanced disease
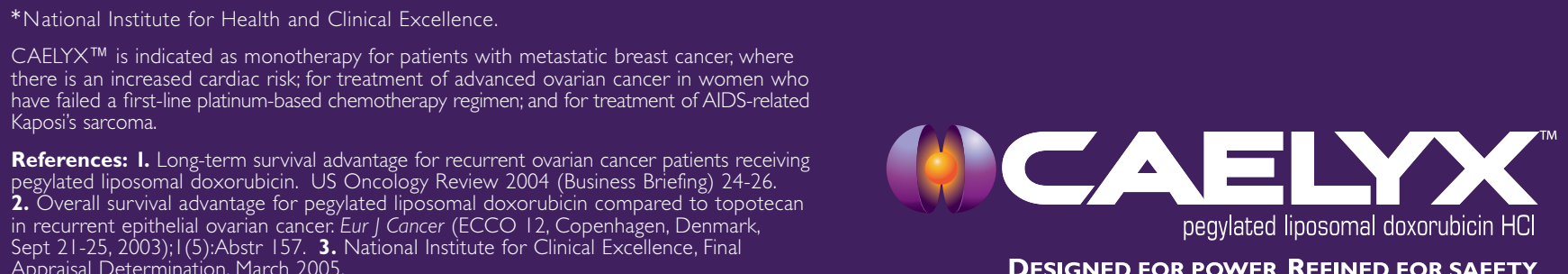

Appraisal Determination, March 2005.

DESIGNED FOR POWER, REFINED FOR SAFETY

ABBREVIATED PRESCRIBING INFORMATION Caelyx $10 \mathrm{ml}$ contains doxorubicin hydrochloride $2 \mathrm{mg} / \mathrm{ml}$ in a liposome formulation for intravenous infusion.

Uses: Caelyx is indicated as monotherapy for patients with metastatic breast cancer, where there is an increased in women who have failed a first-line platinum-based chemotherapy regimen; for treatment of AIDS-related Kaposi's sarcoma (KS) in patients with low CD4 counts $<200 \mathrm{CD}_{4}$ lymphocytes/ $\mathrm{mm}^{3}$ ) and extensive mucocutaneous or visceral disease, as first-line or second line chemotherapy of disease that has progressed with, or in patients intolerant to, prior combination systemic chemotherapy comprising at least two of the following agents: a vinca alkaloid, bleomyc

Dosage: Breast Cancer/Ovarian cancer: Caelyx is administered intravenously at a dose of $50 \mathrm{mg} / \mathrm{m}^{2}$ once every 4 weeks for as long as the disease does not

For doses < $90 \mathrm{mg}$ : dilute Caelyx in $250 \mathrm{ml} 5 \%(50 \mathrm{mg} / \mathrm{ml})$ glucose solution for infusion.

For doses $\geq 90 \mathrm{mg}$ : dilute Caelyx in $500 \mathrm{ml} 5 \%(50 \mathrm{mg} / \mathrm{ml})$ glucose solution for infusion.

To minimize the risk of infusion reactions, the initial dose is administered at a rate no greater than $1 \mathrm{mg} /$ minute. If no infusion reaction is observed, subsequent Caelyx infusions
may be administered over a 60 minute period.

AIDS-related KS: Caelyx should be administered intravenously at $20 \mathrm{mg} / \mathrm{m}^{2}$ every two-three weeks. Intervals shorter than 10 ays should be avoided to prevent drug should last for 2-3 months to achieve a therapeutic response and continued as needed to maintain response.
Contra-indications: Patients with history of hypersensitivity to doxorubicin or other components, or who ar may be ding. Caelyx should not be used in AIDS-KS that cystemic Precautions and Warnings: As with all doxorubicin treatment, patients should undergo frequent ECG monitoring for mator of and monitoring of cardiac function (eg echocardiography should only receive Caelyx if the benefits outweigh potential risk. Infusion-associated reactions may occur-see SPC for details.

Caution is needed in patients who have received prior anthracycline therapy and dosage adjustment may be needblood counts during treatment with Caelyx. Caelyx is not reommended for use in pregnant women. Women child-bearing potential should avoid pregnancy whilst they or their male partners are receiving Caelyx.

The most common undesirable effect reported in breast/ovarian clinical trials palmar-plantar erythrodysesskin (PP), characteris mild PPE infrequently redte skermantions, mostly mild. and nausea were also commonly reported in breast/ovarian cancer patient populations, whereas myelosuppression an cancer patient populations, whereas myelosuppression
(mostly leukopaenia) was the most common side effect in AIDS-KS patients.

Clinically significant laboratory abnormalities were infrequent, with elevated total bilirubin, AST and ALT reported ly significant increases in serum $\mathrm{cr}$ y significant increases in serum creatinine were reported
Most frequent side-effects are myelosuppression, leukopae nia, anaemia, neutropaenia and thrombocytopaenia.
Adjustment of Caelyx dosing interval may be needed in such cases.

Other effects are nausea, asthenia, alopecia, fever diarrhoea, infusion associated reactions, stomatitis, palmarplantar syndrome, oral candidiasis, nausea and vomiting,
weight loss, rash, mouth ulceration, dyspnoea, abdominal pain, allergic reaction, vasodilation, anorexia, glossitis, constipation, paresthesia, pain, skin decolouration, dry skin, pharyngitis, dyspepsia, somnolence and retinitis. Bullous Undesirable effects reported for Calyx-treat patients. cancer patients, were breast pain, leg cramps, oedema, leg oedema, peripheral neuropathy, oral pain, ventricular arrhythmia, folliculitis, bone pain, musculo-skeletal pain, thrombocythemia, cold sores (non-herpetic), fungal infection, epistaxis, upper respiratory tract infection, bullous eruption, dermatitis, erythematous rash, nail disorder, scaly skin, lacrimation, and blurred vision.

Overdosage worsens myelosuppression and patients should be hospitalised for symptomatic treatment of toxic effects. Summary of Product Characteristics (SmPC) should be consulted for further details.

Presentations: $10 \mathrm{ml}$ vial containing $2 \mathrm{mg} / \mathrm{ml}$ doxorubicin hydrochloride; $25 \mathrm{ml}$ vial containing $2 \mathrm{mg} / \mathrm{ml}$ doxorubicin hydrochloride

egal Category: Prescription Only Medicine; Marketing Authorisation Numbers: $10 \mathrm{ml}$ vial:

Marketing Authorisation Holder: S-P Europe, Rue de Stalle 73, Brussels, Belgium.

Date of Revision of text: February 2003

S Schering-Plough 
Table 2: Randomised Trials in Platinum-resistant Ovarian Cancer

\begin{tabular}{|c|c|c|c|}
\hline Study & Author/year & $\mathbf{N}$ & Conclusion \\
\hline Paclitaxel versus Topotecan* & $\begin{array}{l}\text { ten Bokkel Huinink; } \\
\text { J. Clin. Oncol. 1997, Annals } 2004\end{array}$ & 226 & Equivalent activity \\
\hline PLD versus Topotecan & $\begin{array}{l}\text { Gordon; J. Clin. Oncol. 200I, } \\
\text { Gynecol. Oncol. } 2004\end{array}$ & 255 & $\begin{array}{l}\text { Equivalent activity; less } \\
\text { serious toxicity }\end{array}$ \\
\hline PLD versus Paclitaxel & O’Byrne; ASCO 2002 & 131 & $\begin{array}{l}\text { Equivalent activity; } \\
\text { alternative without alopecia, } \\
\text { neuropathy }\end{array}$ \\
\hline $\begin{array}{l}\text { Topotecan qd X } 5 \text { versus } \\
q \text { wk* }^{*}\end{array}$ & Hoskins; J. Clin. Oncol. 1998 & 63 & $\begin{array}{l}\text { Improved activity with daily } \\
\text { administration }\end{array}$ \\
\hline $\begin{array}{l}\text { Paclitaxel q } 3 \text { week versus } \\
q \text { wk* regimen }\end{array}$ & Andersson; ASCO 2000 & 208 & $\begin{array}{l}\text { Equivalent activity; } q \text { wk } \\
\text { has less toxicity }\end{array}$ \\
\hline $\begin{array}{l}\text { Paclitaxel/epirubicin } \\
\text { versus paclitaxel }\end{array}$ & Bolis; Gynecol. Oncol. 1999 & 81 & Equivalent activity \\
\hline
\end{tabular}

*Includes patients with platinum-sensitive disease.

Figure 2: PLD Relative to GCIG

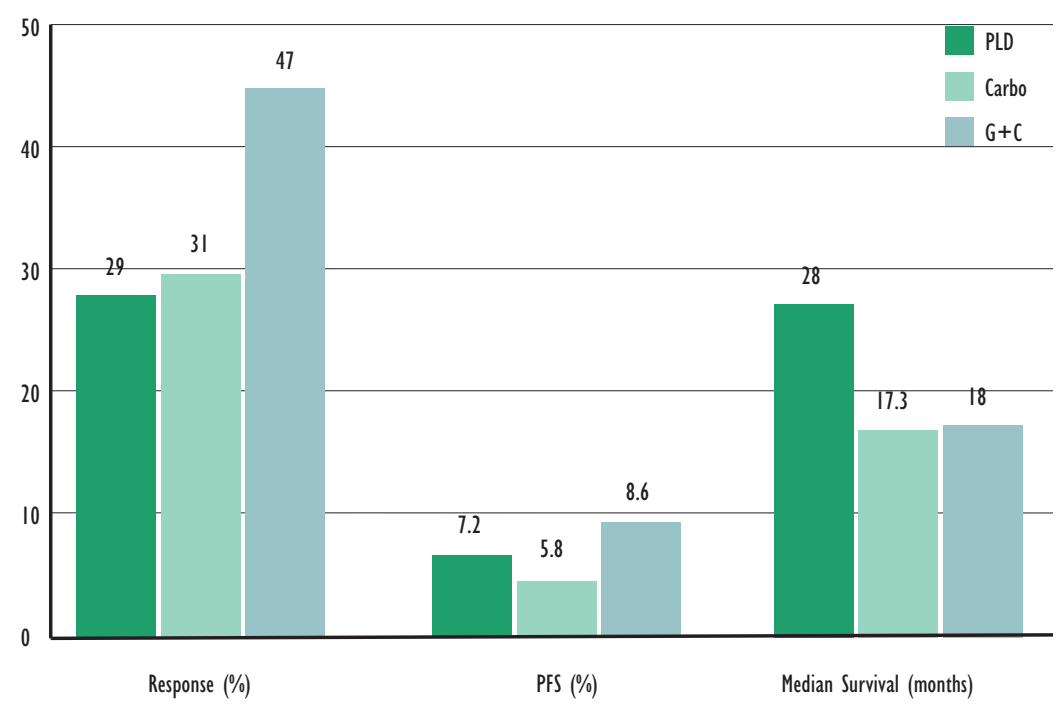

GCIC trial used gemcitabene as the second agent in the combination. While the combination again showed a significantly better response rate and progression-free survival, there was no difference in overall survival.

Does this mean that all patients with platinumsensitive disease should be retreated with combination platinum-based therapy? Although all of these trials were randomised, there are significant demographic differences that may explain some of the apparent differences in the study results (see Table 1).

The ICON 4 study had a greater number of patients who relapsed after 12 months relative to the AGO-GCIC trial, which in turn was greater than that in the trial of PLD versus topotecan. A similar pattern was seen when looking at the number of patients who had not received prior taxane in each study. A single agent would certainly be expected to have a lower response rate than a combination; however, the two-year survival with single agent PLD was similar to that of the platinum-taxane combination in ICON 4 and the median survival was equivalent to the combination (see Figure 1). The response rate of single-agent PLD was equivalent to that of singleagent carboplatin in the AGO trial (see Figure 2). The progression-free survival was superior to single-agent carboplatin and similar to the gemcitabene combination and the median overall survival of single-agent PLD was superior to either arm of the AGO trial.

Those patients who have a prolonged platinumfree interval, will probably have a better progression-free and possibly overall survival with combination therapy; however, for those patients with early platinum-sensitive relapse, i.e. six to 12 months, the data does not clearly indicate an advantage for platinum-based therapy at this time.

In summary, no agent or combination has shown any significant improvement by any measure in platinum-resistant disease. The ease of administration and low toxicity profile would favour the use of PLD as the agent of choice in platinum-resistant disease. PLD is the only nonplatinum agent to demonstrate a progression-free and overall survival advantage in platinum-sensitive disease and should be the first choice whenever resorting to a non-platinum agent.

Although combination therapy has shown improvement on response rates and progressionfree survival in platinum-sensitive patients, it is not clear at this time that equivalent results might not be achieved with sequential single-agent therapy, without the added toxicity. 
European Society of

Gynaecological Oncology

\section{4th INTERNATIONAL MEETING OF THE \\ EUROPEAN SOCIETY OF \\ GYNAECOLOGICAL ONCOLOGY (ESGO)}

ISTANBUL, TURKEY, SEPTEMBER 25.29, 2005

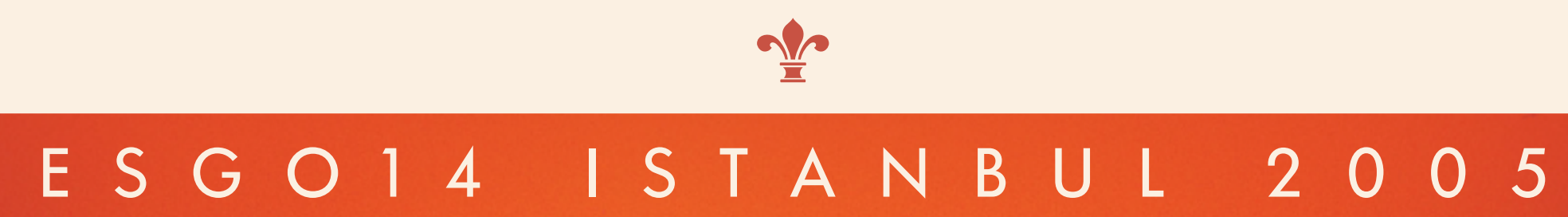

Chairman, ESGO 14

Uzi Beller
Co - Chairman, ESGO 14
Ali Ayhan
ESGO President

Ignace Vergote
Please visit the website for updates, online abstract submission and registration www.esgo.org/esgo 14
Meeting Secretariat - ESGO14

17 rue du Cendrier, P.O. Box 1726, CH-1211 Geneva 1, Switzerland Tel: +41 22908 0488; Fax: +41 22732 2850; E-mail: esgo14@esgo.org 